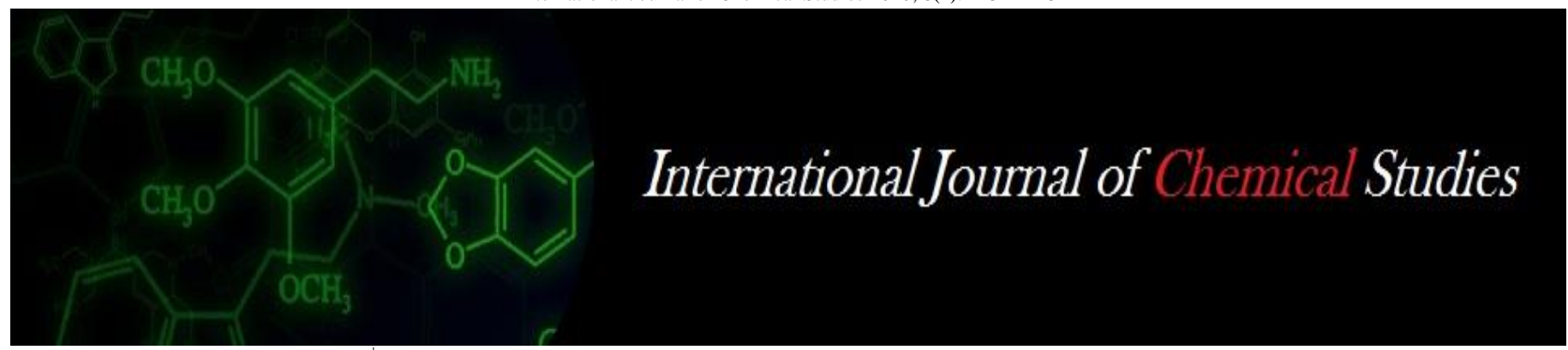

P-ISSN: 2349-8528

E-ISSN: 2321-4902

IJCS 2020; 8(1): 1434-1445

(C) 2020 IJCS

Received: 15-11-2019

Accepted: 21-12-2019

\section{VB Hamane}

Assistant Professor of Plant Pathology, Vivekanand College of Agriculture, Hiwara Bk. Tq. Mehkar Dist. Buldana,

Maharashtra, India

\section{Dr. MS Bhale}

Senior Scientist (Plant

Pathology), Department of Plant

Breeding and Genetics,

Jawaharlal Nehru Krishi Vishwa

Vidyalaya, Jabalpur,

Maharashtra, India

\section{AD Harne}

Senior Research Fellow (Plant Pathology), Centre for Organic Agriculture Research and Training, Dr. Panjabrao Deshmukh Krishi Vidyapeeth, Akola, Maharashtra, India

\section{RR Kale}

Assistant Professor of Plant Pathology, Vivekanand College of Agriculture, Hiwara Bk. Tq. Mehkar Dist. Buldana, Maharashtra, India

\section{Study of mycoflora associated with seeds obtained from farmers as compared to certified seeds}

\author{
VB Hamane, Dr. MS Bhale, AD Harne and RR Kale
}

DOI: https://doi.org/10.22271/chemi.2020.v8.i1t.8459

\begin{abstract}
In India, soybean (Glycine max (L.) Merrill) is a significant legume widely accepted due to high protein and oil content and on account of neutraceutical and pharmaceutical values. The investigation was under taken to determine the seed health status of farmers own saved seeds and certified seeds of soybean, associated mycoflora, its impact on seed sowing quality and management of soybean. During the period 150 seed samples obtained form 09 districts spread over 4 agroclimatic regions were analyzed for the associated mycoflora through Standard blotter method and visual observations on Diaphanoscope.

The seed association of Macrophomina phaseolina, causal agent of charcoal rot ranged from 3.0 to $15.0 \%$ while Colletotrichum dematium, causal agent of anthracnose and pod blight, was in the ranged of 2.0 to $10.0 \%$. The association of Fusarium oxysporum, causal agent of seed root, seedling decay was from 10.0 to $14.0 \%$. The seed rot causing fungi Aspergillus niger (3.0 to 12.0\%) Aspergillus flavus (4.0 to $11.0 \%$ ) were noticed. The purple stain of soybean seed, caused by Cercospora kikuchii was in the ranged of (4.0 to $15.0 \%$ ) and Soybean mosaic virus infected ranged from (1.0 to 4.0\%).
\end{abstract}

Keywords: mycoflora, Macrophomina, Colletotrichum, Fusarium, Aspergillus, Cercospora, Soybean

\section{Introduction}

Soybean (Glycine max (L.) Merrill) a native of eastern Asia is a significant legume widely accepted due to high protein and oil content and on account of neutraceutical and pharmaceutical values. Soybean seeds contain protein $(43 \%)$, oil $(21 \%)$ and are good source of lysine $(6.4 \%)$ which is a limiting factor in cereals. The contribution of India in the world soybean area and production is about 7.9 and 2.3\%, respectively. In India during 2013-2014 the area under soybean was 108.834 lakh hectares with the production of 104.366 lakh tones and productivity being $959 \mathrm{~kg} / \mathrm{ha}$. (Anonymous, 2014) ${ }^{[6]}$.

In Madhya Pradesh the area under soybean cultivation during kharif 2014 was 55.462 lac hectares as compared to 62.605 lac hectares during kharif 2013 showing a decrease of 11.40 per cent. However, in 2013 the productivity was abnormally low and inspite of lower area, the production during kharif 2014 was 60.249 lac $\mathrm{mt}$ as compared to 43.262 lac mt during kharif 2013 showing an increase of 39.26 percent (SOPA, 2014) ${ }^{[34]}$.

Soybean is attacked by a number of pathogens viz., fungi, bacteria, viruses and nematode. Many are seedborne and about 35 diseases are economically important in India (Gupta and Chouhan, 2005) ${ }^{[17]}$. There are about 13 pathogens transmitted through seeds. The seedborne mycoflora are responsible for seed rot and decay with manifestation of various diseases at different growth stage of soybean plant (Bhale, 2004) ${ }^{[13]}$. Annual yield losses by diseases in soybean have been reported to the tune 10-30\% at global level (Hartman et al., 1999) [18]. Seedborne pathogens cause losses in seed quality and quantity. The mycoflora reduces the germination and seedling vigour. Pathogens are responsible for seed rot, seedling blight, root and stem rot, foliar and pod infection (Agarwal and Joshi, 1972; Agarwal et al., 1974) ${ }^{[2,4]}$. A number of seedborne pathogens including Colletotrichum dematium, Macrophomina phaseolina, Fusarium oxysporum, Aspergillus niger, Aspergillus flavus, Phoma medicaginis, Cercospora kikuchii, Myrothecium roridum, Alternaria alternata and Penicillium sp. are reported (Bhale et al., $2004^{[13]}$; Gupta and Chauhan, 2005) ${ }^{[17]}$.

\section{Material and Methods}

The status of mycoflora associated with soybean seeds obtained from farmers of various agroclimatic zones of Madhya Pradesh was determined. The seed belonged to farmers and
Corresponding Author: VB Hamane

Assistant Professor of Plant Pathology, Vivekanand College of Agriculture, Hiwara Bk. Tq Mehkar Dist. Buldana

Maharashtra, India 
certified category. The impact of associated mycoflora on sowing seed quality was investigated. The effectiveness of chemical fungicides and biopesticides against seed borne mycoflora was investigated in the present study. Material used and methods followed are described herewith.

\section{General}

\section{Cleaning and sterilization of apparatus}

The glassware used during the course of investigation was of Corning and Borosil make. Prior to use, each glassware was cleaned with chromic acid solution.

\section{Preparation of chromic acid solution}

Sulphuric acid: $300 \mathrm{ml}$

Potassium dichromate: $80 \mathrm{~g}$

Distilled water: $400 \mathrm{ml}$

The glassware was cleaned with acid solution followed by thorough washing with detergent powder and finally rinsed with normal tap water and/or distilled water as per need. The air dried glassware was sterilized in an autoclave at 1.05 $\mathrm{kg} / \mathrm{cm}^{2}$ (15 lb per square inch) for 15 minutes. Whereas, sand and field soil at $1.05 \mathrm{~kg} / \mathrm{cm}^{2}$ for 180 minutes. Plastic trays were disinfested with $0.1 \%$ Copper sulphate solution and later washed by sterile water.

The inoculation needle, forceps and biological needle were surface disinfested by dipping in alcohol and there after heating over a flame. The inner surface of growth chamber and clean air flow system was disinfested by using exposing the Ultra violet rays through UV lamps and spray of formaldehyde solution. Prior to use, safety precaution were adopted while using ultra violet lamp and formaldehyde solution.

\section{Media}

The ingredients of media used during the course of investigation are as follow:

\section{Potato sucrose agar (PSA)}

Peeled and sliced healthy potato: $200 \mathrm{~g}$

Sucrose: $20 \mathrm{~g}$

Agar-agar: $20 \mathrm{~g}$

Distilled water: $1000 \mathrm{ml}$

\section{Incubation chamber}

The seeded petriplates were incubated under two set of Philips 40 Watt day tube light placed horizontally at the height of $40 \mathrm{~cm}$. Alternate cycles of $12 \mathrm{hr}$ light and $12 \mathrm{hr}$ dark period were maintained.

\section{Collection of seed sample}

Seed samples of soybean variety JS 335 were obtained from farmers of 09 districts covering 4 agroclimatic zones of Madhya Pradesh. Certified seed sample from respective location were also procured and used for comparison. From each district 5 samples of certified seed and 5 samples of farmers own saved seed were obtained through Seed Technology Research Centre, JNKVV, Jabalpur. In all 90 seed samples were obtained. The samples were numbered and stored in paper envelops under low temperature condition to avoid further deterioration. The seeds were tested by dry seed examination and incubation techniques. Collection of farmers own saved and certified seed sample from seven agroclimatic zones covering 09 districts
Detection of mycoflora associated with soybean seeds

\begin{tabular}{|c|c|}
\hline Agroclimatic zone & District \\
\hline Kymore plateau and Satpura Hills & Jabalpur, Katni, Seoni \\
\hline Satpura plateau & Chhindwara, Betul \\
\hline Nimar valley & Khandwa, Khargaon \\
\hline Central Narmada valley & Hoshangabad, Narasinghpur \\
\hline
\end{tabular}

\section{Dry seed examination}

The certified seed samples and samples of farmers own saved seeds were examined on diaphanoscope. The diseased seed samples were sorted and identified on the basis of symptoms.

\section{Standard Blotter method}

Seed collected from different sources were tested by standard blotter method (ISTA, 1996) ${ }^{[21]}$ for the associated mycoflora. Three circular blotter papers of the size of petridishes $(90 \mathrm{~mm})$ were cut and dipped in sterilized water. Excess of water was removed and placed in each sterilized petridish. In each petridish, 10 soybean seeds were placed with the help of presterilized forcep (eight in outer circle and two in the centre). The seeded petridishes were incubated in the growth chamber. Seed were pretreated with $0.1 \% \mathrm{NaoCl}$ for 20 seconds. Petridish were examined on fifth day of incubation. Mycoflora were identified on the basis of colony and habit character, subsequently confirming by making slides of fungal structure, fruiting bodies and spores.

\section{Standard ragdoll method}

Standard Ragdoll method (ISTA, 1996) ${ }^{[21]}$ was used for the testing and effect of the associated mycoflora on germination of soybean seed.

In the method, four hundred seed of each category and sample were used. The towel (blotters) papers were moistened with sterilized water. Excess of water was removed, the paper were stretched over the flat surface and kept over clean surface of the working table. Fifty seed were arranged on the half portion of the towel paper. Seed were covered with the other half portion of the paper and rolled over. A wax paper was wrapped on the rolled paper towel and both ends were tightened with rubber bands. It prevented the run-off of water and helped in maintenance of the moisture required for seed germination process. The rolled towel papers were kept in a slanting position in a plastic tray. The seeded towel were placed in a seed germinator at $25{ }^{\circ} \mathrm{C}$ with $\mathrm{RH}$ around $85 \%$. The seedlings were examined on the $10^{\text {th }}$ day of incubation and germination per cent was calculated.

\section{Standard agar plate method}

In the method, potato sucrose agar medium was used. The medium was transferred in each pre sterilized petridish. With the help of forcep, pretreated seed were placed on 18-20 ml solidified and cooled PSA at equal distance. The seed were placed in a manner so that 8 were in outer ring and two in the center. Observation on the basis of colony and habit character of developing associated mycoflora were recorded on $5^{\text {th }}$ day of incubation. Mycoflora were identified on the basis of developing colony and habits characters and observed directly under stereoscopic binocular microscope. Confirmation of the mycoflora was made by making slides under compound microscope.

\section{Identification of mycoflora}

The mycoflora developing on incubated seeds were identified on the basis of nature of colony characteristics, fruiting bodies and habit character directly under stereoscopic binocular 
microscope and subsequent preparation of microslides. Help of fungal identification key developed by Kulshreshtha et al. (1976), Ahmed and Reddy (1993) [5], Ram Nath et al. (1970) ${ }^{[25]}$, Barnet (1965) ${ }^{[10]}$, Tiffany and Gilman (1954) ${ }^{\text {[35] }}$ and Booth (1971) ${ }^{[14]}$, were used for the purpose.

\section{Impact of seed associated mycoflora On seed germination}

The effect of seed associated mycoflora was determined by Standard Ragdoll method (between the blotters), Standard Blotter method (top of the blotters).

\section{On seed emergence}

In the method counted soybean seeds were sown in a plastic tray filled with sterilized soil and sand kept under laboratory condition. Seed emergence was recorded after 5 and 10 day incubation. The trays were irrigated with sterile water whenever required. Light was provided by horizontally hang day tube light. Seed emergence was also determined under field conditions.

\section{On seedling vigour index}

Seedling vigour index was calculated based on the seedling length as per the formula recommended by Abdul-Baki and Anderson (1973) ${ }^{[1]}$.

Seedling vigour index $=$ (Mean root length + mean shoot length) $\times$ percent seed germination

To determine seedling vigour index, 10 seedling of each sample grown between towel papers were used. Shoot length was measured from the collar region to the point of attachment of cotyledons. Root length was measured from the collar region to the tip of main root.

\section{On viability}

The effect of seed mycoflora on seed viability was determined by tetrazolium salt test.

Reagent: $1 \%$ tetrazolium solution (2, 3, 5- triphenyl tetrazolium chloride)

Procedure: The seed of all the categories were completely immersed in distilled water for $18 \mathrm{hr}$ to initiate activity of dehydrogenase enzyme and to facilitate penetration of tetrazolium solution. The testa (seed coat) of the seed was removed with the help of forcep and then remaining part of the seed immersed in $1 \%$ tetrazolium solution for $3 \mathrm{hr}$ at $20^{0}$ $\mathrm{C}$ in complete dark. Then the seeds were rinsed with water and examined. Each seed was evaluated as viable or dead on the basis of staining pattern and intensity of the red colour.

\section{On seed coat cracking}

Influence of mycoflora on seed coat cracking was recorded by Ferric-Chloride $\left(\mathrm{FeCl}_{3}\right)$ test.

\section{Chemical used: $\mathrm{FeCl}_{3}$ solution $20 \%$}

Procedure: In the test, the selected seed samples of all the categories were soaked in $20 \%$ Ferric chloride solution placed in a beaker. After 15 minutes observation on the development of black stain on the seed surface was investigated. Observations were recorded by naked eye as well the help of hand lense (10x). Qualitative observation was taken.

\section{Management of seed associated mycoflora}

The seeds of soybean pre tested variety JS 335 having maximum natural infection of target pathogens was used. The seeds were treated with individual fungicides and observations were recorded on the associated mycoflora adopting standard blotter method (ISTA, 1996) ${ }^{[21]}$, Standard Ragdoll method (ISTA, 1996) ${ }^{[21]}$

\section{Standard blotter method}

Fungicide treated seeds were used. Untreated seeds served as control. In the method, 3 circular blotter papers of the size of the petridish were cut and dipped in sterilized water. Excess water was removed and soaked sheets were placed in each petridish. Twenty five soybean seeds were placed in each petridish with the help of sterilized forcep under aseptic conditions of inoculation chamber. In the petriplate 16 seeds were placed in outer circle, 8 in the inner circle and 1 in the center so as to allow in the equal distance between the seeds. Seeded plates were kept for the incubation in the chamber. Fungi were identified by making slides and observing under microscope on eight day of incubation with the help of identification manuals.

\section{Dry seed treatment with fungicide and biopesticide}

Each category of soybean seeds was taken in polythene bag and required quantity of fungicide and biopesticide was sprinkled over the seeds. The fungicides and biopesticides seeds were gently shake so as to get uniform coating on individual seed. The treated seeds were sown in sterile soil and sand media at equal distance in a plastic tray. The effect of fungicides and biopesticides on seed germination, seed emergence and seedling vigour was recorded by sowing the treated seeds in sterile soil and sand in a plastic tray. Observation was recorded on 6 and 9 days after sowing.

Table: Fungicides and biopesticides used for seed treatment

\begin{tabular}{|c|c|c|c|}
\hline Fungicide & \% Conc. & Doses/kg seeds & Applications \\
\hline Thiram + Carbendazim & 0.25 & $2.5 \mathrm{~g}$ & Seed treatment \\
\hline Thiram + Carboxin & 0.20 & $2.0 \mathrm{~g}$ & Seed treatment \\
\hline Copper oxychloride & 0.25 & $2.5 \mathrm{~g}$ & Seed treatment \\
\hline Carboxin & 0.20 & $2.0 \mathrm{~g}$ & Seed treatment \\
\hline Carbendazim & 0.20 & $2.0 \mathrm{~g}$ & Seed treatment \\
\hline Tebuconazole & 0.20 & $2.0 \mathrm{~g}$ & Seed treatment \\
\hline Thiride & 0.25 & $2.5 \mathrm{~g}$ & Seed treatment \\
\hline Trichoderma viride & 0.60 & $6.0 \mathrm{~g}$ & Seed treatment \\
\hline Trichoderma harzianum & 0.60 & $6.0 \mathrm{~g}$ & Seed treatment \\
\hline Pseudomonas Fluorescence & 0.60 & $6.0 \mathrm{~g}$ & Seed treatment \\
\hline Untreated control & - & - & - \\
\hline
\end{tabular}

\section{Result and Discussion}

In the present investigation seed health status of farmers own saved seed was determined and compared with certified seed category. The impact of associated mycoflora on sowing seed quality was investigated and the observations on the effectiveness of fungicides and biopesticides were recorded and results are presented herewith.

\section{Collection of seed sample}

In all 90 seed samples of soybean variety JS 335 were obtained from farmers of 09 districts covering 04 agroclimatic zones of Madhya Pradesh (Table 1). The seed sample were numbered and stored in paper envelops under low temperature condition. The seed samples were subjected for seed health by different standard technique (ISTA, 1996) ${ }^{[21]}$. 
Table 1: Sources of soybean seed sample of variety JS 335 from different agroclimatic zones

\begin{tabular}{|c|c|c|c|c|}
\hline Agro climatic Zone & District & Farmer seed & Certified seed & Total \\
\hline \multirow{3}{*}{ Kymore plateau \& Satpura Hills } & Jabalpur & 05 & 05 & 10 \\
\hline & Katni & 05 & 05 & 10 \\
\hline & Seoni & 05 & 05 & 10 \\
\hline \multirow{2}{*}{ Satpura plateau } & Chhindwara & 05 & 05 & 10 \\
\hline & Betul & 05 & 05 & 10 \\
\hline \multirow{2}{*}{ Nimar valley } & Khandwa & 05 & 05 & 10 \\
\hline & Khargoan & 05 & 05 & 10 \\
\hline \multirow{2}{*}{ Central Narmada valley } & Hoshangabad & 05 & 05 & 10 \\
\hline & Narasinghpur & 05 & 05 & 10 \\
\hline 04 & 09 & 45 & 45 & 90 \\
\hline
\end{tabular}

\section{Detection of mycoflora by Standard Blotter method}

Association of mycoflora with soybean seeds obtained from farmer and certified seed samples was detected by standard blotter method (ISTA, 1996) ${ }^{[21]}$. Results of association of mycoflora were detected from the seed samples belonging to 7 agroclimatic zone and 15 district of Madhya Pradesh are presented.

\section{Kymore plateau \& Satpura Hills}

In all, 15 seed samples from 3 district (Jabalpur, Katni and Seoni) were analysed. Data presented in Table 02 indicate the association of 5 major mycoflora in variable proportions. Maximum association of Macrophomina phaseolina (10.0\%) was recorded from the seed samples from Seoni. Seed samples from Katni had shown maximum association of Colletotrichum dematium (6.0\%). Association of Fusarium oxysporum was maximum $(14.0 \%)$ in the seed sample from Seoni and Jabalpur in the farmer seed samples.

It was noticed that association of mycoflora was lesser in certified seed samples as compared to farmer seeds (10.0\%). Incidence of Macrophomina phaseolina was only $4.0 \%$ in certified seed. While Colletotrichum dematium (4.0\%) was recorded as compared to in certified seed. Association of Fusarium oxysporum was $(10.0 \%)$ while Aspergillus flavus was $5.0 \%$ in certified seed. The germination percent ranged up to $77.0 \%$ in farmer seed as compared to $81.0 \%$ in certified seed (Table 02).

Table 2: Association of mycoflora with soybean seeds obtained from farmers of Kymore Plateau \& Satpura Hills and certified seed samples as detected by Standard blotter method (ISTA, 1996) ${ }^{[21]}$

\begin{tabular}{|c|c|c|c|c|c|}
\hline \multirow[b]{2}{*}{$\begin{array}{c}\text { Agro climatic Zone / District / } \\
\text { Sample }\end{array}$} & \multirow[b]{2}{*}{ Mycoflora } & \multicolumn{2}{|c|}{ Farmer seed } & \multicolumn{2}{|c|}{ Certified seed } \\
\hline & & $\begin{array}{c}\text { Percent } \\
\text { association }\end{array}$ & $\begin{array}{l}\text { Percent seed } \\
\text { germination }\end{array}$ & $\begin{array}{c}\text { Percent } \\
\text { association }\end{array}$ & $\begin{array}{l}\text { Percent seed } \\
\text { germination }\end{array}$ \\
\hline \multicolumn{6}{|c|}{ Kymore Plateau \& Satpura Hills } \\
\hline Jabalpur & & & & & \\
\hline \multirow{5}{*}{ Sample 01} & $\begin{array}{l}\text { Macrophomina } \\
\text { phaseolina }\end{array}$ & 2.0 & 77.0 & 3.0 & 81.0 \\
\hline & $\begin{array}{l}\text { Colletotrichum } \\
\text { dematium }\end{array}$ & 5.0 & & 4.0 & \\
\hline & Fusarium oxysporum & 11.0 & & 7.0 & \\
\hline & Aspergillus niger & 7.0 & & 6.0 & \\
\hline & Aspergillus flavus & 7.0 & & 3.0 & \\
\hline \multirow{5}{*}{ Sample 02} & $\begin{array}{l}\text { Macrophomina } \\
\text { phaseolina }\end{array}$ & 1.0 & 75.0 & 0.0 & 81.0 \\
\hline & $\begin{array}{l}\text { Colletotrichum } \\
\text { dematium }\end{array}$ & 3.0 & & 0.0 & \\
\hline & Fusarium oxysporum & 10.0 & & 5.0 & \\
\hline & Aspergillus niger & 6.0 & & 2.0 & \\
\hline & Aspergillus flavus & 9.0 & & 1.0 & \\
\hline \multirow{5}{*}{ Sample 03} & $\begin{array}{l}\text { Macrophomina } \\
\text { phaseolina }\end{array}$ & 2.0 & 70.0 & 0.0 & 80.0 \\
\hline & $\begin{array}{l}\text { Colletotrichum } \\
\text { dematium }\end{array}$ & 5.0 & & 3.0 & \\
\hline & Fusarium oxysporum & 9.0 & & 4.0 & \\
\hline & Aspergillus niger & 7.0 & & 6.0 & \\
\hline & Aspergillus flavus & 0.0 & & 0.0 & \\
\hline \multirow{5}{*}{ Sample 04} & $\begin{array}{l}\text { Macrophomina } \\
\text { phaseolina }\end{array}$ & 2.0 & 72.0 & 0.0 & 73.0 \\
\hline & $\begin{array}{l}\text { Colletotrichum } \\
\text { dematium }\end{array}$ & 5.0 & & 1.0 & \\
\hline & Fusarium oxysporum & 8.0 & & 0.0 & \\
\hline & Aspergillus niger & 7.0 & & 0.0 & \\
\hline & Aspergillus flavus & 6.0 & & 2.0 & \\
\hline \multirow{2}{*}{ Sample 05} & $\begin{array}{l}\text { Macrophomina } \\
\text { phaseolina }\end{array}$ & 5.0 & 75.0 & 2.0 & 75.0 \\
\hline & $\begin{array}{l}\text { Colletotrichum } \\
\text { dematium }\end{array}$ & 5.0 & & 1.0 & \\
\hline
\end{tabular}




\begin{tabular}{|c|c|c|c|c|c|}
\hline & Fusarium oxysporum & 8.0 & & 4.0 & \\
\hline & Aspergillus niger & 3.0 & & 3.0 & \\
\hline & Aspergillus flavus & 5.0 & & 3.0 & \\
\hline \multicolumn{6}{|l|}{ Katni } \\
\hline \multirow{5}{*}{ Sample 01} & $\begin{array}{c}\text { Macrophomina } \\
\text { phaseolina }\end{array}$ & 5.0 & 70.0 & 0.0 & 68.0 \\
\hline & $\begin{array}{c}\text { Colletotrichum } \\
\text { dematium }\end{array}$ & 2.0 & & 1.0 & \\
\hline & Fusarium oxysporum & 7.0 & & 5.0 & \\
\hline & Aspergillus niger & 4.0 & & 2.0 & \\
\hline & Aspergillus flavus & 3.0 & & 3.0 & \\
\hline \multirow{5}{*}{ Sample 02} & $\begin{array}{c}\text { Macrophomina } \\
\text { phaseolina }\end{array}$ & 3.0 & 70.0 & 4.0 & 78.0 \\
\hline & $\begin{array}{c}\text { Colletotrichum } \\
\text { dematium }\end{array}$ & 3.0 & & 3.0 & \\
\hline & Fusarium oxysporum & 6.0 & & 2.0 & \\
\hline & Aspergillus niger & 2.0 & & 1.0 & \\
\hline & Aspergillus flavus & 7.0 & & 5.0 & \\
\hline \multirow{5}{*}{ Sample 03} & $\begin{array}{c}\text { Macrophomina } \\
\text { phaseolina }\end{array}$ & 2.0 & 71.0 & 2.0 & 65.0 \\
\hline & $\begin{array}{c}\text { Colletotrichum } \\
\text { dematium }\end{array}$ & 1.0 & & 1.0 & \\
\hline & Fusarium oxysporum & 6.0 & & 2.0 & \\
\hline & Aspergillus niger & 7.0 & & 7.0 & \\
\hline & Aspergillus flavus & 6.0 & & 3.0 & \\
\hline \multirow{5}{*}{ Sample 04} & $\begin{array}{c}\text { Macrophomina } \\
\text { phaseolina }\end{array}$ & 4.0 & 71.0 & 2.0 & 75.0 \\
\hline & $\begin{array}{c}\text { Colletotrichum } \\
\text { dematium }\end{array}$ & 6.0 & & 2.0 & \\
\hline & Fusarium oxysporum & 5.0 & & 0.0 & \\
\hline & Aspergillus niger & 6.0 & & 0.0 & \\
\hline & Aspergillus flavus & 6.0 & & 0.0 & \\
\hline \multirow{5}{*}{ Sample 05} & $\begin{array}{c}\text { Macrophomina } \\
\text { phaseolina }\end{array}$ & 5.0 & 73.0 & 1.0 & 63.0 \\
\hline & $\begin{array}{c}\text { Colletotrichum } \\
\text { dematium }\end{array}$ & 6.0 & & 2.0 & \\
\hline & Fusarium oxysporum & 4.0 & & 0.0 & \\
\hline & Aspergillus niger & 2.0 & & 1.0 & \\
\hline & Aspergillus flavus & 2.0 & & 0.0 & \\
\hline \multicolumn{6}{|l|}{ Seoni } \\
\hline \multirow{5}{*}{ Sample 01} & $\begin{array}{c}\text { Macrophomina } \\
\text { phaseolina }\end{array}$ & 7.0 & 75.0 & 3.0 & 70.0 \\
\hline & $\begin{array}{c}\text { Colletotrichum } \\
\text { dematium }\end{array}$ & 3.0 & & 2.0 & \\
\hline & Fusarium oxysporum & 10.0 & & 5.0 & \\
\hline & Aspergillus niger & 1.0 & & 0.0 & \\
\hline & Aspergillus flavus & 5.0 & & 0.0 & \\
\hline \multirow{5}{*}{ Sample 02} & $\begin{array}{c}\text { Macrophomina } \\
\text { phaseolina }\end{array}$ & 6.0 & 71.0 & 1.0 & 71.0 \\
\hline & $\begin{array}{c}\text { Colletotrichum } \\
\text { dematium }\end{array}$ & 4.0 & & 0.0 & \\
\hline & Fusarium oxysporum & 12.0 & & 10.0 & \\
\hline & Aspergillus niger & 7.0 & & 2.0 & \\
\hline & Aspergillus flavus & 7.0 & & 3.0 & \\
\hline \multirow{5}{*}{ Sample 03} & $\begin{array}{c}\text { Macrophomina } \\
\text { phaseolina }\end{array}$ & 0.0 & 76.0 & 0.0 & 70.0 \\
\hline & $\begin{array}{c}\text { Colletotrichum } \\
\text { dematium }\end{array}$ & 3.0 & & 1.0 & \\
\hline & Fusarium oxysporum & 12.0 & & 6.0 & \\
\hline & Aspergillus niger & 6.0 & & 3.0 & \\
\hline & Aspergillus flavus & 0.0 & & 1.0 & \\
\hline \multirow{5}{*}{ Sample 04} & $\begin{array}{c}\text { Macrophomina } \\
\text { phaseolina }\end{array}$ & 8.0 & 72.0 & 2.0 & 72.0 \\
\hline & $\begin{array}{c}\text { Colletotrichum } \\
\text { dematium }\end{array}$ & 2.0 & & 0.0 & \\
\hline & Fusarium oxysporum & 14.0 & & 3.0 & \\
\hline & Aspergillus niger & 0.0 & & 4.0 & \\
\hline & Aspergillus flavus & 2.0 & & 5.0 & \\
\hline Sample 05 & $\begin{array}{c}\text { Macrophomina } \\
\text { phaseolina }\end{array}$ & 10.0 & 76.0 & 0.0 & 71.0 \\
\hline
\end{tabular}




\begin{tabular}{|c|c|c|c|c|c|}
\hline & $\begin{array}{c}\text { Colletotrichum } \\
\text { dematium }\end{array}$ & 0.0 & & 1.0 & \\
\hline & Fusarium oxysporum & 12.0 & & 8.0 & \\
\hline & Aspergillus niger & 0.0 & & 0.0 & \\
\hline & Aspergillus flavus & 0.0 & & 2.0 & \\
\hline \multirow{5}{*}{ Maximum Association } & $\begin{array}{l}\text { Macrophomina } \\
\text { phaseolina }\end{array}$ & 10.0 & $70.0-77.0$ & 4.0 & $63.0-81.0$ \\
\hline & $\begin{array}{c}\text { Colletotrichum } \\
\text { dematium }\end{array}$ & 6.0 & & 4.0 & \\
\hline & Fusarium oxysporum & 14.0 & & 10.0 & \\
\hline & Aspergillus niger & 7.0 & & 7.0 & \\
\hline & Aspergillus flavus & 9.0 & & 5.0 & \\
\hline
\end{tabular}

\section{Central Narmada Valley}

In Central Narmada Valley, seed sample from Hoshangabad and Narasinghpur district were analysed. Association of Macrophomina phaseolina was $12.0 \%$ in farmer seed as compared to $6.0 \%$ in certified seed whereas Colletotrichum dematium $5.0 \%$ in farmer seed and $4.0 \%$ in certified seed. In certified seed association of Fusarium oxysporum was $10.0 \%$, Aspergillus niger 5.0\%, Aspergillus flavus $3.0 \%$ as compared to $11.0 \%, 7.0 \%, 5.0 \%$ in farmer seed, respectively (Table 03 ).
Maximum association of Macrophomina phaseolina (12.0\%), $11.0 \%$ Fusarium oxysporum was recorded in the farmer seed samples from Hoshangabad district. The seed germination ranged up to $75.0 \%$ in farmer seed sample while it was $78.0 \%$ in certified seed. In certified seed samples the seed germination was higher and association was lesser as compared to farmer seed (Table 03).

Table 3: Association of mycoflora with soybean seeds obtained from farmers of Central Narmada Valley and certified seed samples as detected by Standard blotter method (ISTA, 1996) ${ }^{[21]}$

\begin{tabular}{|c|c|c|c|c|c|}
\hline \multirow{2}{*}{$\begin{array}{c}\text { Agro climatic Zone / District / } \\
\text { Sample }\end{array}$} & \multirow[b]{2}{*}{ Mycoflora } & \multicolumn{2}{|c|}{ Farmer seed } & \multicolumn{2}{|c|}{ Certified seed } \\
\hline & & $\begin{array}{c}\text { Percent } \\
\text { association }\end{array}$ & $\begin{array}{c}\% \text { seed } \\
\text { germination }\end{array}$ & $\begin{array}{c}\text { Percent } \\
\text { association }\end{array}$ & $\begin{array}{c}\% \text { seed } \\
\text { germination }\end{array}$ \\
\hline \multicolumn{6}{|c|}{ Central Narmada Valley } \\
\hline \multicolumn{6}{|l|}{ Hoshangabad } \\
\hline \multirow{5}{*}{ Sample 01} & $\begin{array}{l}\text { Macrophomina } \\
\text { phaseolina }\end{array}$ & 7.0 & 73.0 & 3.0 & 76.0 \\
\hline & Colletotrichum dematium & 3.0 & & 2.0 & \\
\hline & Fusarium oxysporum & 5.0 & & 4.0 & \\
\hline & Aspergillus niger & 7.0 & & 3.0 & \\
\hline & Aspergillus flavus & 3.0 & & 0.0 & \\
\hline \multirow{5}{*}{ Sample 02} & $\begin{array}{l}\text { Macrophomina } \\
\text { phaseolina }\end{array}$ & 6.0 & 74.0 & 6.0 & 72.0 \\
\hline & Colletotrichum dematium & 2.0 & & 2.0 & \\
\hline & Fusarium oxysporum & 11.0 & & 10.0 & \\
\hline & Aspergillus niger & 4.0 & & 2.0 & \\
\hline & Aspergillus flavus & 2.0 & & 0.0 & \\
\hline \multirow{5}{*}{ Sample 03} & $\begin{array}{l}\text { Macrophomina } \\
\text { phaseolina }\end{array}$ & 11.0 & 73.0 & 5.0 & 75.0 \\
\hline & Colletotrichum dematium & 0.0 & & 0.0 & \\
\hline & \begin{tabular}{|l|} 
Fusarium oxysporum \\
\end{tabular} & 10.0 & & 6.0 & \\
\hline & Aspergillus niger & 5.0 & & 2.0 & \\
\hline & Aspergillus flavus & 5.0 & & 0.0 & \\
\hline \multirow{5}{*}{ Sample 04} & $\begin{array}{l}\text { Macrophomina } \\
\text { phaseolina }\end{array}$ & 12.0 & 75.0 & 6.0 & 73.0 \\
\hline & Colletotrichum dematium & 0.0 & & 4.0 & \\
\hline & Fusarium oxysporum & 10.0 & & 2.0 & \\
\hline & Aspergillus niger & 5.0 & & 2.0 & \\
\hline & Aspergillus flavus & 0.0 & & 0.0 & \\
\hline \multirow{5}{*}{ Sample 05} & $\begin{array}{l}\text { Macrophomina } \\
\text { phaseolina }\end{array}$ & 12.0 & 71.0 & 5.0 & 74.0 \\
\hline & Colletotrichum dematium & 5.0 & & 3.0 & \\
\hline & Fusarium oxysporum & 9.0 & & 2.0 & \\
\hline & Aspergillus niger & 5.0 & & 0.0 & \\
\hline & Aspergillus flavus & 5.0 & & 0.0 & \\
\hline \multicolumn{6}{|l|}{ Narasinghpur } \\
\hline \multirow{5}{*}{ Sample 01} & $\begin{array}{l}\text { Macrophomina } \\
\text { phaseolina }\end{array}$ & 5.0 & 71.0 & 2.0 & 72.0 \\
\hline & Colletotrichum dematium & 3.0 & & 1.0 & \\
\hline & Fusarium oxysporum & 8.0 & & 2.0 & \\
\hline & Aspergillus niger & 3.0 & & 3.0 & \\
\hline & Aspergillus flavus & 2.0 & & 0.0 & \\
\hline Sample 02 & Macrophomina & 4.0 & 70.0 & 4.0 & 75.0 \\
\hline
\end{tabular}




\begin{tabular}{|c|c|c|c|c|c|}
\hline & phaseolina & & & & \\
\hline & Colletotrichum dematium & 3.0 & & 0.0 & \\
\hline & Fusarium oxysporum & 8.0 & & 0.0 & \\
\hline & Aspergillus niger & 2.0 & & 5.0 & \\
\hline & Aspergillus flavus & 1.0 & & 3.0 & \\
\hline \multirow{5}{*}{ Sample 03} & $\begin{array}{c}\text { Macrophomina } \\
\text { phaseolina }\end{array}$ & 9.0 & 71.0 & 6.0 & 76.0 \\
\hline & Colletotrichum dematium & 2.0 & & 2.0 & \\
\hline & Fusarium oxysporum & 7.0 & & 2.0 & \\
\hline & Aspergillus niger & 0.0 & & 2.0 & \\
\hline & Aspergillus flavus & 1.0 & & 0.0 & \\
\hline \multirow{5}{*}{ Sample 04} & $\begin{array}{c}\text { Macrophomina } \\
\text { phaseolina }\end{array}$ & 6.0 & 72.0 & 6.0 & 78.0 \\
\hline & Colletotrichum dematium & 4.0 & & 2.0 & \\
\hline & Fusarium oxysporum & 6.0 & & 2.0 & \\
\hline & Aspergillus niger & 2.0 & & 0.0 & \\
\hline & Aspergillus flavus & 0.0 & & 0.0 & \\
\hline \multirow{5}{*}{ Sample 05} & $\begin{array}{c}\text { Macrophomina } \\
\text { phaseolina }\end{array}$ & 2.0 & 72.0 & 2.0 & 72.0 \\
\hline & Colletotrichum dematium & 4.0 & & 0.0 & \\
\hline & Fusarium oxysporum & 3.0 & & 1.0 & \\
\hline & Aspergillus niger & 1.0 & & 0.0 & \\
\hline & Aspergillus flavus & 0.0 & & 0.0 & \\
\hline \multirow{5}{*}{ Maximum association } & $\begin{array}{c}\text { Macrophomina } \\
\text { phaseolina }\end{array}$ & 12.0 & $70.0-75.0$ & 6.0 & $72.0-78.0$ \\
\hline & Colletotrichum dematium & 5.0 & & 4.0 & \\
\hline & Fusarium oxysporum & 11.0 & & 10.0 & \\
\hline & Aspergillus niger & 7.0 & & 5.0 & \\
\hline & Aspergillus flavus & 5.0 & & 3.0 & \\
\hline
\end{tabular}

\section{Satpura Plateau}

Soybean seed samples obtained from Chhindwara and Betul districts were analysed for seed health. Association of Macrophomina phaseolina was maximum (15.0\%) in the farmer seed sample from Chhindwara district. Fusarium oxysporum $(13.0 \%)$ was also higher in the farmers seed of
Chhindwara. Association of Aspergillus flavus was higher in certified seed as compared to farmer seed indicating the inappropriate threshing and harvesting techniques adopted. The germination percent of farmer seed samples ranged from 63.0 to $75.0 \%$ as compared 65.0 to $73.0 \%$ in the certified seed (Table 04).

Table 4: Association of mycoflora with soybean seeds obtained from farmers of Satpura Plateau and certified seed samples as detected by Standard blotter method (ISTA, 1996) ${ }^{[21]}$

\begin{tabular}{|c|c|c|c|c|c|}
\hline \multirow[b]{2}{*}{$\begin{array}{l}\text { Agro climatic Zone / District / } \\
\text { Sample }\end{array}$} & \multirow[b]{2}{*}{ Mycoflora } & \multicolumn{2}{|c|}{ Farmer seed } & \multicolumn{2}{|c|}{ Certified seed } \\
\hline & & $\begin{array}{c}\text { Percent } \\
\text { association }\end{array}$ & $\begin{array}{c}\% \text { seed } \\
\text { germination }\end{array}$ & $\begin{array}{c}\text { Percent } \\
\text { association }\end{array}$ & $\begin{array}{c}\% \text { seed } \\
\text { germination }\end{array}$ \\
\hline \multicolumn{6}{|c|}{ Satpura Plateau } \\
\hline \multicolumn{6}{|l|}{ Chhindwara } \\
\hline \multirow{5}{*}{ Sample 01} & $\begin{array}{l}\text { Macrophomina } \\
\text { phaseolina }\end{array}$ & 13.0 & 75 & 10.0 & 69.0 \\
\hline & Colletotrichum dematium & 5.0 & & 4.0 & \\
\hline & Fusarium oxysporum & 11.0 & & 6.0 & \\
\hline & Aspergillus niger & 2.0 & & 0.0 & \\
\hline & Aspergillus flavus & 0.0 & & 0.0 & \\
\hline \multirow{5}{*}{ Sample 02} & $\begin{array}{l}\text { Macrophomina } \\
\text { phaseolina }\end{array}$ & 7.0 & 72 & 0.0 & 68.0 \\
\hline & Colletotrichum dematium & 5.0 & & 2.0 & \\
\hline & Fusarium oxysporum & 10.0 & & 5.0 & \\
\hline & Aspergillus niger & 2.0 & & 1.0 & \\
\hline & Aspergillus flavus & 4.0 & & 3.0 & \\
\hline \multirow{5}{*}{ Sample 03} & $\begin{array}{l}\text { Macrophomina } \\
\text { phaseolina }\end{array}$ & 4.0 & 73 & 4.0 & 65.0 \\
\hline & Colletotrichum dematium & 5.0 & & 5.0 & \\
\hline & Fusarium oxysporum & 11.0 & & 10.0 & \\
\hline & Aspergillus niger & 2.0 & & 2.0 & \\
\hline & Aspergillus flavus & 0.0 & & 0.0 & \\
\hline \multirow{5}{*}{ Sample 04} & $\begin{array}{l}\text { Macrophomina } \\
\text { phaseolina }\end{array}$ & 15.0 & 70 & 7.0 & 67.0 \\
\hline & Colletotrichum dematium & 3.0 & & 2.0 & \\
\hline & Fusarium oxysporum & 12.0 & & 11.0 & \\
\hline & Aspergillus niger & 0.0 & & 2.0 & \\
\hline & Aspergillus flavus & 4.0 & & 4.0 & \\
\hline
\end{tabular}




\begin{tabular}{|c|c|c|c|c|c|}
\hline \multirow{5}{*}{ Sample 05} & $\begin{array}{c}\text { Macrophomina } \\
\text { phaseolina }\end{array}$ & 15.0 & 70 & 3.0 & 70.0 \\
\hline & Colletotrichum dematium & 4.0 & & 5.0 & \\
\hline & Fusarium oxysporum & 13.0 & & 6.0 & \\
\hline & Aspergillus niger & 0.0 & & 0.0 & \\
\hline & Aspergillus flavus & 4.0 & & 0.0 & \\
\hline \multicolumn{6}{|l|}{ Betul } \\
\hline \multirow{5}{*}{ Sample 01} & $\begin{array}{c}\text { Macrophomina } \\
\text { phaseolina }\end{array}$ & 7.0 & 67.0 & 4.0 & 73.0 \\
\hline & Colletotrichum dematium & 4.0 & & 4.0 & \\
\hline & \begin{tabular}{|l|} 
Fusarium oxysporum \\
\end{tabular} & 11.0 & & 2.0 & \\
\hline & Aspergillus niger & 2.0 & & 0.0 & \\
\hline & Aspergillus flavus & 4.0 & & 0.0 & \\
\hline \multirow{5}{*}{ Sample 02} & $\begin{array}{c}\text { Macrophomina } \\
\text { phaseolina }\end{array}$ & 9.0 & 65.0 & 6.0 & 69.0 \\
\hline & Colletotrichum dematium & 7.0 & & 3.0 & \\
\hline & Fusarium oxysporum & 9.0 & & 3.0 & \\
\hline & Aspergillus niger & 3.0 & & 4.0 & \\
\hline & Aspergillus flavus & 6.0 & & 0.0 & \\
\hline \multirow{5}{*}{ Sample 03} & $\begin{array}{c}\text { Macrophomina } \\
\text { phaseolina }\end{array}$ & 3.0 & 63.0 & 5.0 & 69.0 \\
\hline & Colletotrichum dematium & 2.0 & & 2.0 & \\
\hline & Fusarium oxysporum & 9.0 & & 4.0 & \\
\hline & Aspergillus niger & 3.0 & & 1.0 & \\
\hline & Aspergillus flavus & 5.0 & & 4.0 & \\
\hline \multirow{5}{*}{ Sample 04} & $\begin{array}{c}\text { Macrophomina } \\
\text { phaseolina }\end{array}$ & 4.0 & 70.0 & 2.0 & 70.0 \\
\hline & Colletotrichum dematium & 3.0 & & 0.0 & \\
\hline & \begin{tabular}{|l|} 
Fusarium oxysporum \\
\end{tabular} & 6.0 & & 0.0 & \\
\hline & Aspergillus niger & 2.0 & & 0.0 & \\
\hline & Aspergillus flavus & 0.0 & & 4.0 & \\
\hline \multirow{5}{*}{ Sample 05} & $\begin{array}{c}\text { Macrophomina } \\
\text { phaseolina }\end{array}$ & 6.0 & 69.0 & 5.0 & 70.0 \\
\hline & Colletotrichum dematium & 3.0 & & 0.0 & \\
\hline & Fusarium oxysporum & 2.0 & & 0.0 & \\
\hline & Aspergillus niger & 2.0 & & 0.0 & \\
\hline & Aspergillus flavus & 11.0 & & 10.0 & \\
\hline \multirow{5}{*}{ Maximum association } & $\begin{array}{c}\text { Macrophomina } \\
\text { phaseolina }\end{array}$ & 15.0 & $63.0-75.0$ & 10.0 & $65.0-73.0$ \\
\hline & Colletotrichum dematium & 7.0 & & 5.0 & \\
\hline & Fusarium oxysporum & 13.0 & & 11.0 & \\
\hline & Aspergillus niger & 3.0 & & 4.0 & \\
\hline & Aspergillus flavus & 11.0 & & 10.0 & \\
\hline
\end{tabular}

\section{Nimar Valley}

Data presented in Table 05 indicate that seed sample obtained from Khandwa and Khargone districts were analysed with maximum association of Macrophomina phaseolina (12.0\%) from the farmer of Khargoan district as compared to $7.0 \%$ in certified seed. Association of Fusarium oxysporum was $11.0 \%$, Colletotrichum dematium $7.0 \%$, Aspergillus niger $12.0 \%$ and Aspergillus flavus $10.0 \%$ in farmer seed sample as compared to 10.0, 6.0 and $7.0 \%$ in certified seed respectively (Table 05).

Table 5: Association of mycoflora with soybean seeds obtained from farmers of Nimar Valley and certified seed samples as detected by Standard blotter method (ISTA, 1996) ${ }^{[21]}$

\begin{tabular}{|c|c|c|c|c|c|}
\hline \multirow[b]{2}{*}{$\begin{array}{l}\text { Agro climatic Zone / District / } \\
\text { Sample }\end{array}$} & \multirow[b]{2}{*}{ Mycoflora } & \multicolumn{2}{|c|}{ Farmer seed } & \multicolumn{2}{|c|}{ Certified seed } \\
\hline & & $\begin{array}{c}\text { Percent } \\
\text { association }\end{array}$ & $\begin{array}{l}\text { Percent seed } \\
\text { germination }\end{array}$ & $\begin{array}{c}\text { Percent } \\
\text { association }\end{array}$ & $\begin{array}{l}\text { Percent seed } \\
\text { germination }\end{array}$ \\
\hline \multicolumn{6}{|c|}{ Nimar Valley } \\
\hline \multicolumn{6}{|l|}{ Khandwa } \\
\hline \multirow{5}{*}{ Sample 01} & $\begin{array}{l}\text { Macrophomina } \\
\text { phaseolina }\end{array}$ & 8.0 & 73.0 & 3.0 & 75.0 \\
\hline & $\begin{array}{l}\text { Colletotrichum } \\
\text { dematium }\end{array}$ & 3.0 & & 2.0 & \\
\hline & Fusarium oxysporum & 11.0 & & 7.0 & \\
\hline & Aspergillus niger & 12.0 & & 10.0 & \\
\hline & Aspergillus flavus & 10.0 & & 5.0 & \\
\hline \multirow{2}{*}{ Sample 02} & $\begin{array}{l}\text { Macrophomina } \\
\text { phaseolina }\end{array}$ & 7.0 & 78.0 & 2.0 & 75.0 \\
\hline & $\begin{array}{l}\text { Colletotrichum } \\
\text { dematium }\end{array}$ & 7.0 & & 2.0 & \\
\hline
\end{tabular}




\begin{tabular}{|c|c|c|c|c|c|}
\hline & Fusarium oxysporum & 3.0 & & 5.0 & \\
\hline & Aspergillus niger & 10.0 & & 7.0 & \\
\hline & Aspergillus flavus & 10.0 & & 7.0 & \\
\hline \multirow{5}{*}{ Sample 03} & $\begin{array}{c}\text { Macrophomina } \\
\text { phaseolina }\end{array}$ & 6.0 & 75.0 & 3.0 & 72.0 \\
\hline & $\begin{array}{c}\text { Colletotrichum } \\
\text { dematium }\end{array}$ & 7.0 & & 3.0 & \\
\hline & Fusarium oxysporum & 10.0 & & 7.0 & \\
\hline & Aspergillus niger & 8.0 & & 2.0 & \\
\hline & Aspergillus flavus & 8.0 & & 4.0 & \\
\hline \multirow{5}{*}{ Sample 04} & $\begin{array}{c}\text { Macrophomina } \\
\text { phaseolina }\end{array}$ & 3.0 & 76.0 & 2.0 & 72.0 \\
\hline & $\begin{array}{c}\text { Colletotrichum } \\
\text { dematium }\end{array}$ & 6.0 & & 3.0 & \\
\hline & Fusarium oxysporum & 10.0 & & 10.0 & \\
\hline & Aspergillus niger & 3.0 & & 4.0 & \\
\hline & Aspergillus flavus & 5.0 & & 0.0 & \\
\hline \multirow{5}{*}{ Sample 05} & $\begin{array}{c}\text { Macrophomina } \\
\text { phaseolina }\end{array}$ & 5.0 & 77.0 & 0.0 & 77.0 \\
\hline & $\begin{array}{c}\text { Colletotrichum } \\
\text { dematium }\end{array}$ & 6.0 & & 2.0 & \\
\hline & Fusarium oxysporum & 9.0 & & 0.0 & \\
\hline & Aspergillus niger & 5.0 & & 0.0 & \\
\hline & Aspergillus flavus & 2.0 & & 0.0 & \\
\hline \multicolumn{6}{|l|}{ Khargone } \\
\hline \multirow{5}{*}{ Sample 01} & $\begin{array}{c}\text { Macrophomina } \\
\text { phaseolina }\end{array}$ & 4.0 & 72.0 & 5.0 & 71.0 \\
\hline & $\begin{array}{c}\text { Colletotrichum } \\
\text { dematium }\end{array}$ & 5.0 & & 4.0 & \\
\hline & Fusarium oxysporum & 9.0 & & 3.0 & \\
\hline & Aspergillus niger & 2.0 & & 0.0 & \\
\hline & Aspergillus flavus & 0.0 & & 0.0 & \\
\hline \multirow{5}{*}{ Sample 02} & $\begin{array}{c}\text { Macrophomina } \\
\text { phaseolina }\end{array}$ & 7.0 & 73.0 & 7.0 & 72.0 \\
\hline & $\begin{array}{c}\text { Colletotrichum } \\
\text { dematium }\end{array}$ & 3.0 & & 3.0 & \\
\hline & Fusarium oxysporum & 7.0 & & 5.0 & \\
\hline & Aspergillus niger & 0.0 & & 2.0 & \\
\hline & Aspergillus flavus & 5.0 & & 0.0 & \\
\hline \multirow{5}{*}{ Sample 03} & $\begin{array}{c}\text { Macrophomina } \\
\text { phaseolina }\end{array}$ & 12.0 & 75.0 & 5.0 & 72.0 \\
\hline & $\begin{array}{c}\text { Colletotrichum } \\
\text { dematium }\end{array}$ & 3.0 & & 6.0 & \\
\hline & Fusarium oxysporum & 4.0 & & 2.0 & \\
\hline & Aspergillus niger & 0.0 & & 2.0 & \\
\hline & Aspergillus flavus & 4.0 & & 4.0 & \\
\hline \multirow{5}{*}{ Sample 04} & $\begin{array}{c}\text { Macrophomina } \\
\text { phaseolina }\end{array}$ & 11.0 & 74.0 & 4.0 & 72.0 \\
\hline & $\begin{array}{c}\text { Colletotrichum } \\
\text { dematium }\end{array}$ & 0.0 & & 5.0 & \\
\hline & Fusarium oxysporum & 7.0 & & 0.0 & \\
\hline & Aspergillus niger & 0.0 & & 0.0 & \\
\hline & Aspergillus flavus & 7.0 & & 2.0 & \\
\hline \multirow{5}{*}{ Sample 05} & $\begin{array}{c}\text { Macrophomina } \\
\text { phaseolina }\end{array}$ & 12.0 & 73.0 & 5.0 & 73.0 \\
\hline & $\begin{array}{c}\text { Colletotrichum } \\
\text { dematium }\end{array}$ & 4.0 & & 0.0 & \\
\hline & Fusarium oxysporum & 3.0 & & 2.0 & \\
\hline & Aspergillus niger & 0.0 & & 0.0 & \\
\hline & Aspergillus flavus & 6.0 & & 0.0 & \\
\hline \multirow{5}{*}{ Maximum association } & $\begin{array}{c}\text { Macrophomina } \\
\text { phaseolina }\end{array}$ & 12.0 & $72.0-78.0$ & 7.0 & $71.0-77.0$ \\
\hline & $\begin{array}{c}\text { Colletotrichum } \\
\text { dematium }\end{array}$ & 7.0 & & 6.0 & \\
\hline & Fusarium oxysporum & 11.0 & & 10.0 & \\
\hline & Aspergillus niger & 12.0 & & 10.0 & \\
\hline & Aspergillus flavus & 10.0 & & 7.0 & \\
\hline
\end{tabular}


Seed samples obtained from 04 agroclimatic conditions were tested for the associated mycoflora

The results of association of mycoflora with soybean seed samples are summarized in Table 06. Among 4 agroclimatic zone, incidence of Macrophomina phaseolina was maximum up to $15.0 \%$ in farmer seed sample obtained from Satpura plateau while in certified seed samples. The incidence of
Macrophomina phaseolina was comparatively low (10.0\%) obtained from Satpura plateau as compared to farmer seed. In farmer seed samples Fusarium oxysporum was maximum up to $14.0 \%$ in seed samples from Kymore plateau and Satpura Hills as compared to the certified seed sample from Satpura plateau $(11.0 \%)$.

Table 6: Association of mycoflora with soybean seeds obtained from farmers seven agroclimatic zones and certified seed samples as detected by Standard blotter method (ISTA, 1996) ${ }^{[21]}$

\begin{tabular}{|c|c|c|c|c|c|c|c|c|}
\hline \multirow{3}{*}{ Agro climatic Zone /District / Sample } & \multirow{2}{*}{\multicolumn{3}{|c|}{$\begin{array}{c}\text { Farmer seed } \\
\text { Per cent association }\end{array}$}} & \multirow{3}{*}{$\%$ seed Germination } & \multirow{2}{*}{\multicolumn{3}{|c|}{$\begin{array}{c}\text { Certified seed } \\
\text { Per cent association }\end{array}$}} & \multirow{3}{*}{$\%$ seed germination } \\
\hline & & & & & & & & \\
\hline & Mp & $\mathbf{C d}$ & Fo & & Mp & Cd & Fo & \\
\hline \multicolumn{9}{|c|}{ Kymore plateau \& Satpura Hills } \\
\hline & 10.0 & 6.0 & 14.0 & $70.0-77.0$ & 4.0 & 4.0 & 10.0 & $63.0-81.0$ \\
\hline \multicolumn{9}{|c|}{ Satpura plateau } \\
\hline & 15.0 & 7.0 & 13.0 & $63.0-75.0$ & 10.0 & 5.0 & 11.0 & $65.0-73.0$ \\
\hline \multicolumn{9}{|c|}{ Nimar valley } \\
\hline & 12.0 & 7.0 & 11.0 & $72.0-78.0$ & 7.0 & 6.0 & 10.0 & $71.0-77.0$ \\
\hline \multicolumn{9}{|c|}{ Central Narmada valley } \\
\hline & 12.0 & 5.0 & 11.0 & $70.0-75.0$ & 6.0 & 4.0 & 10.0 & $72.0-78.0$ \\
\hline
\end{tabular}

The present investigation was undertaken to determine the seed health status of farmers own saved seed of soybean and its management. Certified seeds were also collected to determine the difference with farmers seed. Globally about 130 diseases have been reported affecting the soybean crop at various stages of crop growth (Hartman et al., 1999) ${ }^{[18]}$, of which 35 diseases are economically important in India. There are about 13 diseases that are transmitted through seeds.

Several pathogens have been observed that are responsible for causing diseases of soybean (Hartman et al., $1999^{[18]}$ ) and many of these are reported to be transmitted through seeds (Kilpatric, 1952; Wu et al., 1964; Hussain et al., 1989; Poharkar, 1992; Vishwadhar and Sarbhoy, 1987) [23, 38, 19, 29, 31]. Number of the pathogens have been observed on seed (Singh and Thapliyal, 1999; Arya et al., 2004; Begum et al., 2007; Shovan et al., 2008) ${ }^{[33,8,11,32]}$.

Investigations were made to determine the current status of seed mycoflora associated with seed obtained from farmers own saved seed sample as compared to certified seeds. Seed samples of soybean variety JS 335 were procured from the farmers of 09 districts covering 4 agroclimatic zones of Madhya Pradesh. Certified seed sample from respective location were also procured for comparison. From each district 5 samples of certified seed and 5 samples of farmers own saved seed were obtained through Seed Technology Research Centre, JNKVV, Jabalpur.

A number of mycoflora have been observed on soybean seeds. A good deal of recording has been made from Madhya Pradesh, India and global level. Incidence of mycoflora in variable proportions was noticed, however, the incidence of major mycoflora was comparatively less in certified seeds as compared to farmers own saved seeds. Major mycoflora found associated with soybean seeds were Macrophomina phaseolina, Colletotrichum dematium, Fusarium oxysporum, Aspergillus flavus, Aspergillus niger and Cercospora kikuchii. Standard Blotter method was reported to be superior over Standard agar plate method for detection of associated mycoflora by Agarwal et al. (1972), Tripathi and Singh (1991), Vishunawat (2003) [3, 36, 37] also recorded its suitability.

The incidence of seed and seedling diseases have been recorded at caused maximum reduction of seed germination
(Hypperly et al., 1983) ${ }^{[20]}$. The seed samples having natural infection of Macrophomina phaseolina were tested for seed germination by top of the paper and between the blotter paper method. In the sample having no infection, Macrophomina phaseolina has resulted in 89 and $95 \%$ maximum seed germination in top of the paper and between the blotter paper. Seed having maximum infection of Macrophomina phaseolina had shown $72 \%$ germination in top of the paper and $70 \%$ in between the blotter paper. The seed samples having no infection of Colletotrichum dematium obtained from Hoshangabad district have exhibited maximum $91 \%$ germination in top of the paper method and $95 \%$ in between the blotter paper method. Seed sample from Sagar having association of $10.0 \%$ Colletotrichum dematium resulted in 96 to $75 \%$ germination as compared to 75 to $80 \%$ in between the blotter paper method.

Influence of target pathogens on the seed emergence was investigated through sowing of seeds in sterile and unsterile soil and sand. Reduction of $12 \%$ in seed emergence was noticed when seeds were sown in unsterile soil and infected with Macrophomina phaseolina. Whereas $7 \%$ reduction due to Colletotrichum dematium and $5 \%$ due to Fusarium oxysporum was noticed. Mortality was higher in sterile soil, indicating the influence of the particular mycoflora.

Seed samples having no infection of Fusarium oxysporum, Macrophomina phaseolina and Colletotrichum dematium resulted in better seed germination as compared to seed sample having variable infection of the selected mycoflora reduction in seed germination was recorded due to seed infection. Pre and post emergence losses have been reported by Chauhan and Gupta (2005) ${ }^{[17]}$; Anuja et al. (2000) ${ }^{[7]}$.

Results indicate that there has been significant role of seed mycoflora associated with seed of soybean that caused seed and seedling diseases (Hartman et al., $1999^{[18]}$, Bhale et al., 2003) ${ }^{[12]}$.

In the present investigation, influence of seed treatment with biopesticide and chemical fungicide was determine in selected seed samples. In seed samples having maximum infection Macrophomina phaseolina (15.0\%) elimination was observed in seed treated with Copper oxychloride, Carboxin. The association of Macrophomina phaseolina was 1 to $3 \%$ in fungicide treated seed as compared to untreated seed $15.0 \%$. 
Association of Colletotrichum dematium was 1 to $3 \%$ in fungicide treated seed as compared to $9 \%$ in untreated seed. Complete elimination of Colletotrichum dematium was recorded in seeds treated with Thiram + Carbendazim, Thiram +Carboxin, Copper oxychloride, Carboxin, Carbendazim, Tebuconazole, Thiride.

In untreated seed association of Fusarium oxysporum was $14 \%$ while it ranges from 1 to $3 \%$ in treated seed. Association of Fusarium oxysporum was higher in seed treated with biopesticide as compared to chemical fungicide.

\section{References}

1. Abdul-Baki AA, Anderson JD. Vigour determination in soybean seeds of multiple criteri. Crop Science. 1973; 13:630-633.

2. Agarwal VK, Joshi AB. A preliminary note on the purple stain disease of soybean. Indian Phytopathology. 1972; 24(4):810-812.

3. Agarwal VK, Mathur SB, Neergaard P. Some aspects of seed health testing with respect to seed borne fungi of rice, wheat, black gram, green gram and soybean grown. Indian Phytopathology. 1972; 25:91-100.

4. Agarwal VK, Singh OV, Nene YL. Influence of fungicidal seed treatment on the mycoflora of stored seed and seedling emergence. Indian Journal Agriculture Science. 1974; 43(8):820-824.

5. Ahmed KM, Reddy R. A pictorial guide to the identification of seed borne fungi of sorghum, pearl millet, finger millet, chickpea, pigeonpea and groundnut. Information bulletin No. 34, Pantencheru, India. International Crop Research Institute for the Semi Arid Tropics, 1993, 200.

6. Anonymous. Directorate of Economics and Statistics Department of Agriculture and Cooperation, Goverment of India, New Delhi, 2014.

7. Anuja, Gupta KR, Aneja, Gupta A. Field efficacy of seed dressing chemicals on seedling emergence. Seed yield and seed weight in soybean Seed Research. 2000; 28(1):54-58.

8. Arya VK, Vishunavat K, Negi H. Detection location and transmission of seed borne inoculums of Macrophomina phaseolina in charcoal rot in soybean. Journal of Mycology \& Plant Pathology. 2004; 34(2):233-237.

9. Baker D. Apron seed treatment on soybean. Special circular Ohio Agricultural. Research and Development Center. 2001; 179:9-10.

10. Barnet HL. Illustrate genera of imperfect fungi. Burgress Publication Company, Minneopolis, USA, 1965.

11. Begum MM, Sariah M, Putech AB, Zainal MA, Abidin. Detection of seed borne fungi and site of infection by Collectotrichum truncatum in naturally infected soybean seeds. International Journal of Agricultural Research. 2007; 2:812-819.

12. Bhale MS, Khare D, Sharma ND, Varma RK. On the spot diagnosis and identification of major seed borne diseases of soybean. Manual DRS/02/2003, JNKVV, Jabalpur, 2003, 125p.

13. Bhale MS. Mycoflora associated with soybean seeds, detection and their management. Journal Mycopatholagical Research. 2004; 42(1):27-29.

14. Booth C. The genus Fusarium, kew surrey, UK Commonwealth Mycological Institute, UK, 1971, 273p.

15. Gaikwad SJ, Goharkar RT, Meshram SU. Occurrence of pod blight of soybean caused by Colletotrichum dematium, Journal of Maharashtra Agriculture University. 1993; 18(3):489-491.

16. Goulart ACP, Andrade PIM, Borges EP. Control of soybean seed borne pathogens by fungicide treatment and its effect on emergence and yield. Summa Phytopathologica. 2000; 26(3):341-346.

17. Gupta GK, Chouhan SS. Symptoms, identification and management of soybean diseases. Techinical Bulletin. National Research Centre for Soybean (ICAR), Khandwa Road, Indore, India, 2005, 92p.

18. Hartman GL, Sinclair JB, Rupe JC. Compendium of soybean diseases (4th edition). The American Phytopathological Society, APS Press, St. Paul, Min., USA, 1999, 100p.

19. Hussain S, Hussain S, Khan BA. Seed borne mycoflora of soybean in the Northwest Frontier province of Pakistan. Sarhad Journal of Agriculture. 1989; 3(4):421424.

20. Hypperly PR, Mignucci JS, Sinclair JB, Mandoza JB. Seed Science \& Technology. 1983; 11:371-380.

21. ISTA. International Rules for seed testing. Seed Science \& Technology 21(Supplement), Switzerland, 1996, 286p.

22. Khan SU. studies on leaf spot disease of soybean (Glycine $\max$ (L.) Merrill) incited by Myrothecium roridum Tobe ex Friesia M. Sc. (Ag.) Thesis, JNKVV, Jabalpur (MP), 1992.

23. Kilpatric RA. Fungi associated with soybean seeds and pods, prior to harvest at stoneville, Mississipi in 1952. Phytopathology. 1952; 42:292.

24. Malhotre S. Studies on fungal pathogens associated with soybean seed and their control. M. Sc. (Ag.) Thesis, JNKVV, Jabalpur (MP), 1997.

25. Nath R, Neergard P, Mathur SB. Identification of Fusarium species as they occur in blotter tests. Proceeding of the International Seed Testing Association. 1970; 35(1):121-144.

26. Nene YL, Thapliya PN. Fungicides in plant disease control, Oxford and IBH, New Delhi, 1993, 507p.

27. Pandit A. Integrated control of collar rot (Sclerotium rolfsii Sacc.) and dry root rot (Rhizoctonia bataticola (Taub.) Butler) of soybean. M. Sc. (Ag.) Thesis, JNKVV, Jabalpur (MP), 1992.

28. Patel BL. Studies on aerial blight of soybean (Glycine $\max (\mathrm{L}$.) Merrill) incited by Rhizoctonia solani Khun. M. Sc. (Ag.) Thesis, JNKVV, Jabalpur (MP), 1994.

29. Poharkar MS. Effect of seed treatment on seed borne disease of soybean. Pesticides. 1992; 10(3):160-162.

30. Prasanth PS, Patil PV. Seed stain of soybean its incidence, effect on seed quality and integrated managment. Indian Phytopathology. 2007; 60(4):482488.

31. Sarbhoy AK, Vishwadhar P. Soyben rust in India and its management; Farming System and Integrated Management (Eds. Verma JP and Verma A). Malhotra Publishing House, New Delhi -64, India, 1992, 117-120p.

32. Shovan LR, Bhuiyan A, Suitana N, Begum JA, Pervez Z. Prevalence of fungi associated with soybean seeds and pathogencity tests of the major seed borne pathogen. International Journal of Sustainable Crop Production. 2008; 3(4):24-33.

33. Singh U, Thapliyal PN. Fungi responsible for seedling emergence problem in different soybean cultivars in Tarai region. Indian Phytopathology. 1999; 52(1):79-81.

34. SOPA. Statistical data, 2014. http://www.sopa.org 
35. Tiffany LH, Gilman JC. Species of Colletotrichum from legumes. Mycologia. 1954; 46:52-75.

36. Tripathi DP, Singh BR. Mycoflora of soybean seed and their control. Madras Agriculture Journal. 1991; 78(114):130-132.

37. Vishnuwat K. Charcoal rot of soybean in on the spot diagnosis and identification of major seed borne diseases of soybean. Training manual number DRS/02/2003, Department of Plant Breeding and Genetics, JNKVV, Jabalpur, 2003, 55-59p.

38. Wu LC, Lin YS, Chiu KY. Seed-borne diseased soybean in Thiwan II survey of seed-borne pathogens from soybean seeds. Botanical Bulletin Academia Sinica Taipai. 1964; 5(2):105-112. 